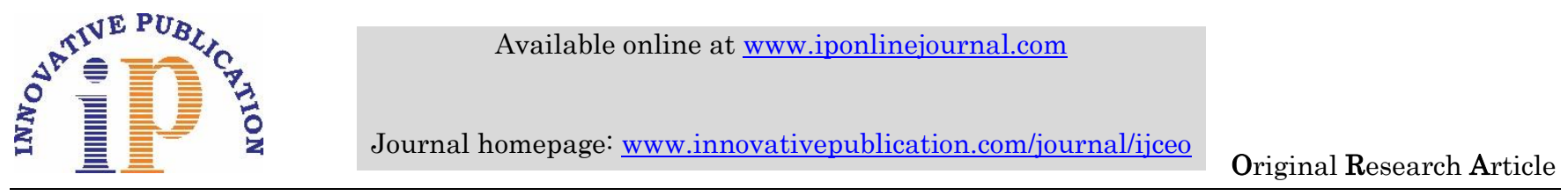

\title{
To evaluate the effect of intravitreal bevacizumab on intraocular pressure
}

\author{
Ashwini G ${ }^{1 *}$, Maheshwar. $\mathbf{N}^{2}$ \\ ${ }^{1}$ Senior Resident, ${ }^{2}$ Assistant Professor, Dept. of Ophthalmology, Chamarajanagar Institute of Medical Sciences (CIMS), Yadapura, \\ Karnataka, India
}

\section{Article Info}

Received: 23 ${ }^{\text {rd }}$ December, 2017

Accepted: $23^{\text {rd }}$ May, 2019

Published Online: $9^{\text {th }}$ September, 2019

Keywords: Bevacizumab, Clinically significant macular edema, Diabetic macular edema, GAT, IOP.

\begin{abstract}
Introduction: To assess intraocular pressure variation after intravitreal bevacizumab injection in patients with diabetic macular edema.

Materials and Methods: Our prospective study included 20 eyes of patients who received intravitreal injection of $0.05 \mathrm{ml}$ bevacizumab as treatment option for diabetic macular edema. IOP was recorded before the injection, at 3 days, 1 week, 1 month and 2 months after the intravitreal injection.

Results: The mean intraocular pressure before injection was $17.28 \pm 3.04 \mathrm{mmHg}$. The mean IOP post bevacizumab at 3 days, 1 week, 1 month and 2 months were $19.98 \pm 3.23 \mathrm{mmHg}$, $18.99 \pm 3.67 \mathrm{mmHg}, 17.31 \pm 3.19$ and $17.15 \pm 3.10$ respectively. There was statistically significant elevation in IOP at 3 days post bevacizumab injection, which reinstated to baseline levels at final follow-up.

Conclusion: Our study indicate that intravitreal injection of bevacizumab is associated with short term IOP elevation.
\end{abstract}

\section{Introduction}

Intravitreal anti-VEGF agents are considered as renowned treatment option for various retinal pathologies and met with considerable success in treating diabetic retinopathy, retinal vein occlusion and age related macular degeneration.

Bevacizumab has been accepted globally as a treatment option for the above mentioned sight threatening diseases. Bevacizumab is a recombinant humanized monoclonal $\operatorname{IgG}$ antibody. It prevents binding of all VEGF-A isoforms to endothelial cell receptors, thereby inhibiting angiogenesis and vascular permeability. Bevacizumab is considered safe in terms of local adverse event safety profile. ${ }^{1,2}$ However few adverse effects have been documented after intravitreal bevacizumab. The addition of intravitreal drug into vitreous cavity is presumed to cause immediate rise in intraocular pressure. Various studies have described transitory, shortterm elevation in IOP post intravitreal anti-VEGF injections. Fewer studies have recorded persisting, long-term elevation in IOP after injection of intravitreal anti-VEGF agents.

Our study intends to assess variation in intraocular pressure after intravitreal injection of bevacizumab.

\section{Materials and Methods}

We conducted a prospective interventional study to assess intraocular pressure changes post intravitreal bevacizumab injection in patients with clinically significant macular edema.

\section{Participants}

We included twenty patients with clinically significant macular edema in our study. Patients with active ocular inflammation, glaucoma, previous history of anti-VEGF injections were not included in the study. The approval from institutional ethical committee and written consent from the patients was obtained after elucidation of procedure and possible side effects.

Goldmann applanation tonometer was employed to record intraocular pressure (IOP) in all the patients by a single investigator during morning hours to reduce the bias of time based variation of intraocular pressure. After proper sterilization of the GAT head, the patient was seated in front of the slit lamp. The eye to be examined was anesthetized with topical anesthetic eye drops. The tear film was stained using flourescein strip and IOP was measured in sitting position. A total of three readings were taken and the average of 3 readings was recorded as baseline IOP. Bevacizumab was injected into vitreous at a dose of $1.25 \mathrm{mg}$ in $0.05 \mathrm{ml}$ under sterile conditions in the operating room. Bevacizumab was injected into vitreous cavity using a straight scleral technique with $30 \mathrm{G}$ needle. All patients were reviewed at 3 days, 7 days, 1 month and 2 months after bevacizumab injection. We reviewed the patients up to 2 months after intravitreal bevacizumab. The study protocol, patient information sheet, and consent form were approved by ethics committee.

\footnotetext{
*Corresponding Author: Ashwini G, Senior Resident, Dept. of Ophthalmology, Chamarajanagar Institute of Medical Sciences (CIMS), Yadapura, Karnataka, India

Email: dr.ashwinig@gmail.com

http://doi.org/10.18231/j.ijceo.2019.066
} 


\section{Results}

\section{Statistical Analysis}

Table 1: Mean intraocular pressure at each follow- up

\begin{tabular}{|c|c|}
\hline Follow- up & Mean IOP \\
\hline Baseline & $17.28 \pm 3.04$ \\
\hline Day 3 & $19.98 \pm 3.23$ \\
\hline 1 week & $18.99 \pm 3.67$ \\
\hline 1 month & $17.31 \pm 3.19$ \\
\hline 2 months & $17.15 \pm 3.10$ \\
\hline
\end{tabular}

Fig. 1: Mean IOP at each follow- up

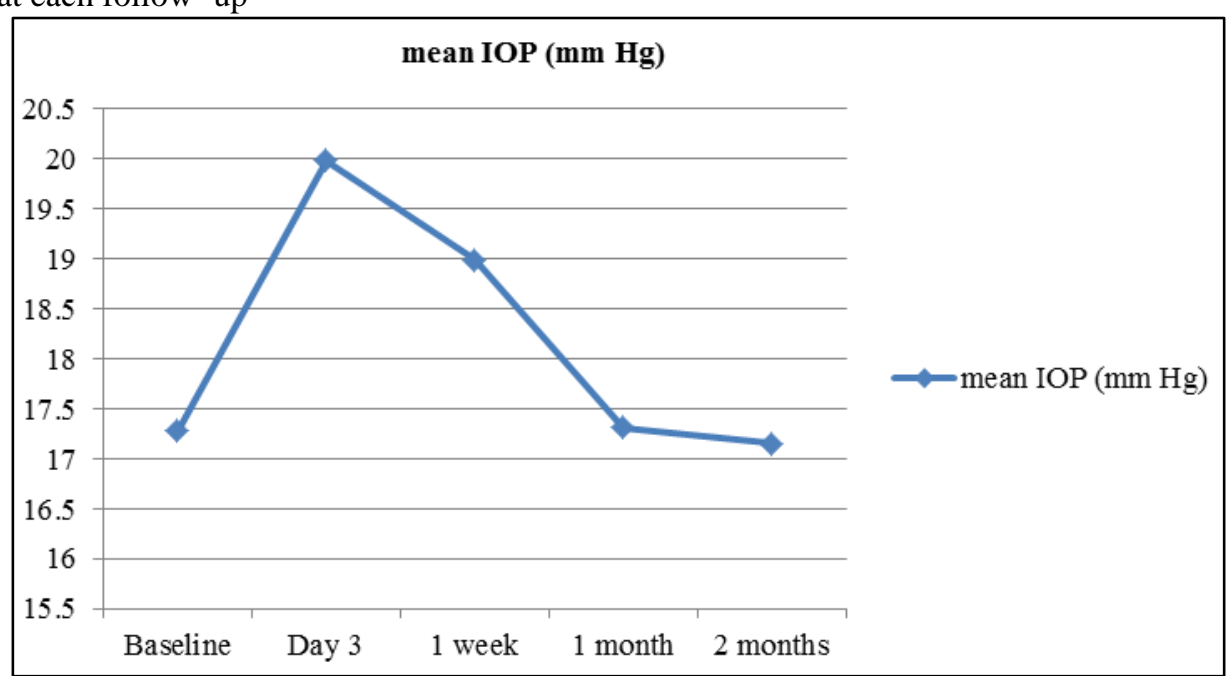

Table 2: Comparison of pre and post- injection intraocular pressure in patients

\begin{tabular}{|c|c|c|c|c|c|c|c|c|}
\hline & $\begin{array}{c}\text { Pre- } \\
\text { treatment }\end{array}$ & 3 Days & $\begin{array}{c}\text { Pre- } \\
\text { treatment }\end{array}$ & 1 week & $\begin{array}{c}\text { Pre- } \\
\text { treatment }\end{array}$ & $\begin{array}{c}\text { 1 } \\
\text { month }\end{array}$ & $\begin{array}{c}\text { Pre- } \\
\text { treatment }\end{array}$ & $\begin{array}{c}\mathbf{2} \\
\text { months }\end{array}$ \\
\hline Mean & 17.28 & 19.98 & 17.28 & 18.99 & 17.28 & 17.31 & 17.28 & 17.15 \\
\hline SD & 3.04 & 3.23 & 3.04 & 3.67 & 3.04 & 3.19 & 3.04 & 3.10 \\
\hline t-value & \multicolumn{2}{|c|}{-2.714} & \multicolumn{2}{|c|}{-1.597} & \multicolumn{2}{c|}{-0.030} & \multicolumn{2}{c|}{0.138} \\
\hline p-value & \multicolumn{2}{|c|}{0.118} & \multicolumn{2}{c|}{0.975} & \multicolumn{2}{c|}{0.89} \\
\hline
\end{tabular}

Differences between pre and post treatment statistics were assessed using paired t-test. Out of 20 patients it was found that $18(90 \%)$ were males and $2(10 \%)$ were females. The mean age was $55.2 \pm 9.33 \mathrm{yrs}$.

\section{Change of IOP}

Variation in intraocular pressure after intravitreal bevacizumab injection at each follow-up are illustrated in Table 1 and Fig. 1. We noted significant elevation in intraocular pressure at 3 days post intravitreal bevacizumab injection, which returned to baseline levels at 2 months. Comparison of pre and post- injection intraocular pressure at each follow-up is illustrated in Table 2. None of our patients required treatment to control IOP.

\section{Discussion}

Diabetes mellitus remains the most important cause of vision impairment in people of working age. It is estimated that $25 \%$ of people with diabetes mellitus have some degree of diabetic retinopathy and 2- $10 \%$ of people have diabetic macular edema. ${ }^{3}$ Duration of diabetes is considered as a prime risk factor in development of diabetic retinopathy. According to ETDRS study, clinically significant macular edema is a form of diabetic macular edema, which is defined as 1 . Any retinal thickening within $500 \mu \mathrm{m}$ of the foveal center. 2. Hard exudates within $500 \mu \mathrm{m}$ of the foveal center that is associated with adjacent retinal thickening. 3. An area of retinal thickening at least 1 disc area in size, any part of which is located within 1 disc area of the foveal center. Clinically significant macular edema is the foremost cause of vision deterioration in patients with diabetes mellitus. Expeditious diagnosis and scrupulous treatment plays an imperative role in preventing menace of visual impairment in diabetic patients. Varying treatment options are available to intercept progression of diabetic macular edema. Anti- VEGF agents are considered effective in treating patients having diabetic macular edema. Bevacizumab is a recombinant humanized immunoglobulin that inhibits all isoforms of VEGF-A. It tends to increase 
intraocular pressure in patients who undergo intravitreal bevacizumab injection. We included twenty patients in our study that underwent intravitreal bevacizumab injection to address diabetic macular edema. We noted significant elevation in IOP at first visit, which restored to baseline levels at 2 months. The study illustrated early and transitory IOP elevation subsequent to bevacizumab injection. Several reports have documented elevation of IOP subsequent to intravitreal anti-VEGF drugs. Bakri SJ, Moshfeghi DM, Francom $\mathrm{S}^{4}$ et al. conducted a study to elucidate IOP variations in patients with age- related macular degeneration who underwent intravitreal ranibizumab injections. Their study presumed that ranibizumab injection is not associated with sustained IOP elevation over 24 months and IOP monitoring is mandatory in patients receiving ranibizumab injections. Unlike our study they have included patients with ARMD, who required monthly ranibizumab injections and reviewed them for 2 years. Dr. Aref ${ }^{5}$ proposed brief and persistent elevation in IOP associated with anti- VEGF agents in his study. According to his study increase in intraocular fluid volume resulted in short term IOP elevation immediately following intravitreal injection. $\mathrm{He}$ also observed that long term IOP elevation was a result of damage to trabecular meshwork, direct pharmacological toxicity and chronic trabecular inflammation. Mazzulla DA, Hariprasad SM, Jager $\mathrm{RD}^{6}$ et al. devised a retrospective study design to delineate IOP fluctuation in patients subjected to intravitreal bevacizumab injection. They reviewed 29 patients who underwent successive intravitreal bevacizumab injections and unveiled significant IOP elevation immediately after the injection, which reinstated to baseline levels within a week. Their study reported that bevacizumab injection of is considered to be safe in accordance with the outlook of short-term IOP elevation. Similar to our study they have illustrated early IOP elevation after bevacizumab injection. Unlike our study they have observed the normalization of IOP by 1 week. Nariani A, Williams B, Hariprasad $\mathrm{SM}^{7}$ performed a retrospective study to elucidate persistent IOP elevation in patients with diabetic macular edema and age related macular degeneration, who were treated with intravitreal anti-VEGF agents. They have reported that injection of anti-VEGF agents was not a considerable risk factor in instigating IOP elevation. Unlike our study this was a retrospective study to discern elevation in IOP subsequent to anti-VEGF injections. Similar to our study they illustrated negligible IOP elevation secondary to anti-VEGF injections in their study design. Several studies have outlined protracted IOP elevation and suggested several presumptions related to risk factors associated with continued IOP elevation. According to these studies rise in IOP is connected with preexisting glaucoma, ocular hypertension and number of anti-VEGF injections. Don Nguyen, Anhtuan Nguyen, Kundandeep $\mathrm{Nagi}^{8}$ et al. designed a retrospective study to analyse longstanding effects of bevacizumab injection on IOP in patients with diabetic macular edema. This study included 100 patients with mean injections ranging from 1 to 3 with a follow- up period of 1 year. The study concluded that there was no statistically significant elevation in IOP in those who received bevacizumab intravitreally, though there is persistent rise in IOP in glaucomatous patients. The study also elucidated minimal correlation between IOP elevation and number of intravitreal injections. Yannuzzi NA, Patel $\mathrm{SN}$, Bhavsar $\mathrm{KV}^{9}$ et al. conducted a cross sectional survey to identify the causative factors leading to persistent elevation of IOP by recruiting neovascular AMD patients. They were treated with intravitreal anti-VEGF injections. Their survey speculated that injection of anti-VEGF agents resulted in trabecular meshwork damage leading to persistent elevation of IOP. Wen JC, Reina- Torres E, Sherwood JM ${ }^{10}$ et al. conducted a study which included patients with age related macular degeneration treated with intravitreal anti-VEGF agents. They observed significant curtailment in outflow facility in patients who received multiple injections of anti-VEGF drugs. Sulman Jaffar, Ali Tyyab, Amena Masrur ${ }^{11}$ et al. conducted a study to determine the impact of Bevacizumab injection on IOP. Their study reported significant elevation in intraocular pressure subsequent to intravitreal bevacizumab injection. The rise in IOP was noted one week after the injection. Similar to our study they have also demonstrated early and transient IOP elevation after intravitreal bevacizumab. Adelman RA, Zheng Q, Mayer $\mathrm{HR}^{12}$ et al. performed a retrospective study to evaluate the role of intravitreal bevacizumab and ranibizumab injections in causing persistent IOP elevation in patients with age related macular degeneration. According to their study $3.45 \%$ of patients developed relentless elevation in IOP secondary to multiple injections of anti-VEGF agents and concluded that sustained IOP elevation can be observed subsequent to repeated intravitreal anti-VEGF injections in patients with unremarkable ocular history. Unlike our study they have administered multiple injections of bevacizumab and they have attributed the sustained elevation of IOP to multiple bevacizumab injections. Wehrli SJ, Tawse K, Levin $\mathrm{MH}^{13}$ et al. conducted a retrospective study to evaluate IOP elevation rate following intravitreal injections of ranibizumab and bevacizumab in patients with age related macular degeneration. This study elucidated low incidence of delayed IOP elevation in these cases and refuted the role of multiple intravitreal anti-VEGF injections in elevating IOP. Frenkel MP, Haji SA, Frenkel $\mathrm{RE}^{14}$ conducted a study to demonstrate the efficacy of prophylactic IOP lowering agents to prevent pressure spikes subsequent to intravitreal injections of ranibizumab, pegaptanib and bevacizumab. Their study recruited seventy one patients with age related macular degeneration. They were subjected to intravitreal anti-VEGF drugs and recorded significant IOP elevation immediately after the injection. The study demonstrated the inefficacy of prophylactic IOP lowering drugs in preventing post injection IOP spikes secondary to intravitreal injections of ranibizumab, pegaptanib and bevacizumab. Various studies have elucidated the causative role of variation in intravitreal injection techniques in elevating IOP post intravitreal injections. A Study conducted by Hohn F, Mirshali $\mathrm{A}^{15}$ et al. noted significant rise in IOP in patients 
who received tunneled scleral injection. Our study revealed no significant association between lens status and IOP elevation after the injection and there was no association between IOP elevation and diabetic retinopathy progression. Our study emphasizes the importance of baseline IOP measurement in all patients who undergo intravitreal antiVEGF injections and need for close monitoring of these cases. There is an indispensable need of further investigations to elucidate the mechanism of IOP elevation to prevent the undesired complications associated with it.

\section{Conclusion}

Though many studies revealed the effect of intravitreal antiVEGF drugs in causing IOP elevation, we can take imperative steps to safeguard our patients.

It is desirable to obtain baseline IOP and optic nerve status before considering anti-VEGF injection in all cases and it is prudent to assess patients risk for developing glaucoma.

\section{Source of Funding: None.}

\section{Conflict of Interest: None.}

\section{References}

1. Rich RM, Rosenfeld PJ, Puliafito CA. short- term safety and efficacy of intravitreal bevacizumab for neovascular agerelated macular degeneration. Retina 2006;26:495-511.

2. Fung AE, Rosenfeld PJ, Reichel E. The international intravitreal bevacizumab safety survey; using the internet to assess drug safety world wide. Br J Ophthalmol 2006;90:13449.

3. Williams R, Airey M, Baxter H, Forrester J, Kennedy- Martin T, Epidemiology of diabetic retinopathy and macular edema: A systematic review. Eye 2004;18:963-83.
4. Bakri SJ, Moshfeghi DM, Francom S, Rundle AC. Intraocular pressure in eyes receiving monthly ranibizumab in 2 pivotal age- related macular degeneration clinical trials. Ophthalmol 2014;121(5):1102-8.

5. Aref AA. Curr Opin Ophthalmol 2012;23(2):105-10.

6. Mazzulla DA, Hariprasad SM, Jager RD, Mieler WF. Retin Cases Brief Rep 2008;2(3):234-5.

7. Nariani A, Williams B, Hariprasad SM. Indian J Ophthalmol 2016;64(9):643-7.

8. Nguyen D, Nguyen A, Nagi K, Abedi G. Pressure Elevation After Intravitreal Bevacizumab Injections in Patients with Diabetic Macular Edema. Invest Ophthalmol Visual Sci 2014;55:1940.

9. Yannuzzi NA, Patel SN, Bhavsar KV, Sugiguchi F, Freund KB. Am J Ophthalmol 2014;158(2):319-327.

10. Wen JC, Reina- Torres E, Sherwood JM, Challa P, Liu KC Invest Ophthalmol Vis Sci 2017;58(3):1893-8.

11. Jaffar S, T Ali, Masrur A, Majeed A. Effect of Intravitreal Injection of Bevacizumab on Intraocular Pressure. $J$ Rawalpindi Med Coll (JRMC) 2013;17(1):71-4.

12. Adelman RA, Zheng Q, Mayer HR. Persistent ocular hypertension following Intravitreal bevacizumab and ranibizumab injections. Jocul Pharmacol Ther 2010;26(1):105-10.

13. Wehrli SJ, Tawse K, Levin MH, Zaidi A, Pistilli M. A lack of delayed Intraocular pressure elevation in patients treated with Intravitreal injection of bevacizumab and ranibizumab. Retina 2012;32(7):1295-301

14. Frenkel MP, Haji SA, Frenkel RE. Effect of prophylactic intraocular pressure- lowering medication on intraocular pressure spikes after intravitreal injection. Arch Ophthalmol 2010;128(12):1523-7.

15. Hohn F, Mirshali A. Impact of injection techniques on intraocular pressure increase after intravitreal ranibizumab application. Graefes Arch Clin Exp Ophthalmol 2010;248(10):1371-5.

How to cite this article: Ashwini G, Maheshwar. N, To evaluate the effect of intravitreal bevacizumab on intraocular pressure. Indian J Clin Exp Ophthalmol 2019;5(3):275-8. 